\title{
Denise Coussy, Cent romans-monde
}

\section{Elena Pessini}

\section{(2) OpenEdition}

\section{Journals}

\section{Édition électronique}

URL : http://journals.openedition.org/studifrancesi/639

DOI : 10.4000/studifrancesi.639

ISSN : 2427-5856

\section{Éditeur}

Rosenberg \& Sellier

\section{Édition imprimée}

Date de publication : 1 avril 2015

Pagination : 201-202

ISSN : 0039-2944

\section{Référence électronique}

Elena Pessini, « Denise Coussy, Cent romans-monde », Studi Francesi [En ligne], 175 (LIX | I) | 2015, mis en ligne le 01 avril 2015, consulté le 18 septembre 2020. URL : http://journals.openedition.org/ studifrancesi/639; DOI : https://doi.org/10.4000/studifrancesi.639

\section{Ce document a été généré automatiquement le 18 septembre 2020}

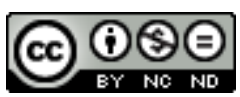

Studi Francesi è distribuita con Licenza Creative Commons Attribuzione - Non commerciale - Non opere derivate 4.0 Internazionale. 


\title{
Denise Coussy, Cent romans-monde
}

\author{
Elena Pessini
}

\section{RÉFÉRENCE}

DENISE COUSSY, Cent romans-monde, Paris, Karthala, 2013, pp. 208.

1 Le désormais célèbre «Manifeste pour une littérature-monde», signé par 44 écrivains et paru en 2007 dans les pages du quotidien «Le Monde», a fait couler beaucoup d'encre. Que l'on partage ou non les positions des signataires, il faut reconnaître que ce texte a eu le mérite de susciter dans l'univers francophone un débat riche et stimulant. L'ouvrage de Denise Coussy se situe, selon ses propres propos, «délibérément dans le sillage des écrits de Michel Le Bris» (p. 9); le titre inscrit sans équivoque possible la démarche de Coussy en étroite parenté avec les travaux de Michel Le Bris qui, après avoir été l'inspirateur du manifeste, a publié deux ouvrages: Pour une littérature-monde (2007) et Je est un autre (pour une identité-monde) (2010), au sein desquels de nombreux écrivains prennent la parole pour se situer dans la vaste mosaïque de la littératuremonde. En utilisant le modèle de cette nouvelle étiquette, sans doute mieux adaptée aux transformations de l'époque contemporaine, Denise Coussy s'attache à dresser un grand inventaire des romans qui pourraient être classés dans cette catégorie. L'analyse ne se focalise pas seulement sur les romans écrits en langue française mais inclut également la production romanesque anglophone et lusophone et étend le domaine de la recherche dans un à rebours qui remonte jusqu'aux premiers récits des grands voyageurs. À défaut de donner une définition précise de ce qu'elle entend par romanmonde, opération délicate qui prêterait sans doute à bien des critiques, Denise Coussy opère un recensement des romans qui témoignent «de l'émergence d'une littérature mondiale à l'œuvre dans les plus vastes continents comme dans les plus petites îles, [d']une nouvelle histoire littéraire de l'humanité où se crée un dialogue des cultures, une communauté de souffrances et finalement une permanence de l'espoir en la liberté» (p. 6). Dans une première partie qui sert de préambule, «Les grands récits de voyage à la découverte du monde» (pp. 11-29), ces derniers sont examinés en tant que source d'inspiration pour les grandes narrations contemporaines. C'est le cas pour Les 
Luciades du portugais Luis Vas de Camoes qui sont à l'origine du Retour des caravelles (1990) d'Antonio Lobo Antunes, pour Le Voyage vers le pôle Sud et autour du monde de James Cook qui a inspiré Le Retour (2007) de la néerlandaise Anna Enquist, pour l'odyssée de l'écossais Mongo Park, Voyage au centre de l'Afrique qui a nourri en 1981 le Water Music de l'anglais T.C. Boyle. La seconde partie, «Le romancier-monde au contact avec les cinq continents» (pp. 29-66) est centrée sur le $\mathrm{xx}^{\mathrm{e}}$ et le tout début du $\mathrm{xxI}^{\mathrm{e}}$ siècles pour montrer, dans un premier chapitre, comment le contact avec l'Afrique, la rencontre avec le continent africain, a fait naître un florilège de récits depuis Conrad en passant par André Gide, Albert Londres, Michel Leiris, Georges Ballandier pour arriver jusqu'à Le Clézio et Jean-Christophe Rufin. Le découpage de cette deuxième partie est bien géographique puisque, après l'Afrique, le second chapitre «Les romanciers et le tout-monde», permet d'élargir le regard sur des horizons plus vastes, comme l'Inde et la Patagonie. Ce sont surtout les œuvres d'écrivains anglophones comme Kipling, Chatwin, Naipaul et Kunsu qui sont examinées, même si Denise Coussy consacre aussi son analyse aux ouvrages de Nicolas Bouvier et d'Henri Michaux. Dans la troisième partie, «Le héros-monde» (pp. 67-128), s'esquissent et se précisent les traits des personnages qui, d'un roman à l'autre, d'une langue à l'autre, s'affirment sur le modèle d'une typologie qui se fait écho et établit des parentés et des affinités. Les quatre parties qui composent ce troisième volet proposent les figures centrales des romans-monde tels que nous les a présentés Denise Coussy. Avant tout la figure de l'esclave. Selon une modalité et une méthodologie adoptées pour l'ensemble de ce travail critique, le spécialiste dresse un inventaire, en mélangeant textes francophones, lusophones et anglophones, des narrations qui mettent en scène les figures d'esclaves, qu'ils soient en captivité ou en situation de révolte et de marronnage. Le lecteur et le chercheur reconnaissent immédiatement ceux qui peuvent être considérés désormais comme des classiques et faisant partie d'un "canon" de la littérature postcoloniale et/ ou de la littérature-monde: Texaco, La Mulâtresse Solitude, L'Esclave vieil homme et le Molosse, mais figurent aussi dans l'inventaire des textes moins connus, moins étudiés qui éveilleront sans doute la curiosité et l'intérêt. Particulièrement digne d'attention est la démarche de certains écrivains dont: «L'obsession de l'esclavage a conduit [...] à proposer des récits-fictions basés sur une inversion hypothétique et parodique des rapports de force» (p. 84). L'exploité et l'exilé constituent deux autres récurrences des romans-monde car, comme le souligne Denise Coussy: «Dès les années cinquante, on voit donc émerger la volonté de certains écrivains engagés de proposer une littérature militante qui donnerait la parole aux exploités du tout-monde» (p. 95). Revers de la médaille de l'exil, le retour au pays natal auquel est consacré le quatrième et dernier chapitre de ce troisième volet de l'enquête, un leitmotiv que l'on retrouve dans les titres mêmes des œuvres. Depuis, bien sûr, le Cahier d'un retour au pays natal de Césaire jusqu'au Fleuve détourné de Rachid Mimouni et au très récent L'Énigme du retour de Dany Laferrière. Dans le quatrième volet de l'ouvrage, «Le Roman-monde» (pp. 129-168), on quitte le point de vue des personnages pour établir une série de dénominateurs communs qui unissent toutes ces narrations, nées parfois aux antipodes les unes des autres. Le titre des différents chapitres qui charpentent cette partie de l'étude permettent aisément d'approcher l'analyse: «Revisiter les romans classiques», «Passer d'une culture à l'autre», "Créer des "patries" imaginaires». L'emploi du canon traditionnel occidental pour en opérer une refonte et une réactualisation à partir d'un point de vue tout à fait nouveau est un fil rouge qui unit bon nombre de romansmonde, tout comme la question de la langue d'écriture, langue empruntée, imposée, 
revisitée, choisie, revendiquée. Le dernier chapitre donne plusieurs exemples de romans-mondes où «beaucoup de romanciers [ont été amenés] à créer carrément des patries "imaginaires" qui leur permettraient de proposer des représentations sublimées de la réalité» (p. 154). Le premier d'entre eux qui fait l'objet d'une analyse, même s'il ne rentre pas stricto sensu dans l'aire linguistique annoncée au début du travail, étant bien entendu Cent ans de solitude de Gabriel Garcia Marquez. Dans la cinquième et dernière partie, «Écritures-monde» (pp. 169-197), l'accent est mis sur les choix esthétiques des auteurs en matière de langage, sur les choix opérés par les écrivains-monde pour libérer la langue qu'ils emploient dans leurs textes des carcans de la tradition. Une grande place est laissée en clôture de l'essai à l'œuvre d'Édouard Glissant, «car nul autre écrivain n'est parvenu à mieux mettre en images ce concept de tout-monde que l'écrivain martiniquais a inlassablement proposé et affiné tout au long de ses essais» (p. 190).

2 L'ampleur du corpus examiné (cent romans-monde) donne parfois à ce travail critique l'apparence d'un inventaire qui aurait peut-être gagné à être moins ambitieux; toutefois le parcours tracé par Denise Coussy permet de suivre la genèse et l'affirmation de ce qui pourrait être considéré comme un genre littéraire à part entière. 